\title{
Évaluation de l'impact du projet Lifebox au Burkina Faso sur la pratique de l'oxymétrie pulsée et la check-list de l'organisation mondiale de la santé pour la sécurité du patient au bloc opératoire
}

\author{
Cheik Tidiane Hafid Wind-Pouiré Bougouma, MD (1) • Nazinigouba Ouédraogo, MD • \\ Rawéléguinbasba Armel Flavien Kaboré, MD · Kélan Bertille Ki, MD • \\ Ibrahim Alain Traoré, MD • Sié Ahmed Ouattara, MD • Gérard Zongo, MD • \\ Martin Lankoandé, MD • Salah Idriss Seif Traoré, MD
}

Received: 19 June 2018/Revised: 13 August 2018/Accepted: 13 August 2018/Published online: 27 November 2018

(c) Canadian Anesthesiologists' Society 2018

Au rédacteur en chef,

La fondation Lifebox, la Société d'anesthésieréanimation et de médecine d'urgence du Burkina, la « World Federation of Societies of Anaesthesiologists » et la «Canadian Anaesthesiologists' Society International Education Foundation » avaient mis en œuvre en 2013 le projet Lifebox au Burkina Faso. Ce projet avait permis la formation de 118 agents anesthésistes sur la pratique de l'oxymétrie pulsée et la check-list de l'Organisation Mondiale de la Santé (OMS) pour la sécurité du patient au bloc opératoire, ainsi que la distribution de 136 oxymètres de pouls à 57 hôpitaux publics.

Nous avons mené une étude pour évaluer l'impact de ce projet sur la pratique de l'oxymétrie pulsée et de la check-list une année après sa mise en œuvre.

Il s'est agi d'une étude descriptive dont la collecte des données a été réalisée par enquête téléphonique du 07 août au 11 septembre 2014 auprès de responsables anesthésistes des 57 hôpitaux bénéficiaires du projet Lifebox. Les

Cette lettre est accompagnée d'un éditorial. Veuillez vous référer à : Can J Anesth 2018; 65 : ce numéro.

C. T. H. W.-P. Bougouma, MD $(\bowtie) \cdot$ R. A. F. Kaboré, MD .

S. A. Ouattara, MD

Centre Hospitalier Universitaire de Tingandogo, Tingandogo,

Burkina Faso

e-mail: chekymed29@yahoo.fr

N. Ouédraogo, MD

Centre Hospitalier Universitaire Yalgado Ouédraogo,

Ouagadougou, Burkina Faso

K. B. Ki, MD

Centre Hospitalier Universitaire Pédiatrique Charles De Gaulle,

Ouagadougou, Burkina Faso données avaient été analysées grâce au logiciel EPI INFO dans sa version 3.5.1 ( Center for Disease Control and Prevention » en collaboration avec l'OMS, Atlanta, GA, USA) et le risque $\propto$ a été fixé à $5 \%$.

Le taux de participation à l'étude était de $100 \%$. Deux (3\%) médecins anesthésiste-réanimateurs et 55 (97\%) infirmiers spécialistes en anesthésie-réanimation ont été interrogés. Avant le projet Lifebox, 95 oxymètres fonctionnels étaient disponibles pour 136 salles d'intervention. Vingt-huit (49 \%) blocs opératoires avaient un oxymètre de pouls fonctionnel dans chaque salle d'opération. L'oxymétrie pulsée était pratiquée systématiquement dans 42 (73 \%) hôpitaux au cours de l'anesthésie. Seuls 10 (17\%) hôpitaux avaient une salle de surveillance post interventionnelle (SSPI) fonctionnelle et pour tous ces SSPI, l'oxymètre utilisé pour la surveillance des patients provenait de la salle d'opération. La check-list de l'OMS était pratiquée dans 10 (17\%) hôpitaux.

Après le projet Lifebox, l'oxymétrie pulsée était pratiquée systématiquement dans tous les hôpitaux au cours de l'anesthésie et elle était pratiquée pour la surveillance du réveil des patients dans 54 (94\%)

\author{
I. A. Traoré, MD \\ Centre Hospitalier Universitaire Souro Sanou, Bobo-Dioulasso, \\ Burkina Faso \\ G. Zongo, MD \\ Centre Hospitalier Régional Universitaire de Ouahigouya, \\ Ouahigouya, Burkina Faso \\ M. Lankoandé, MD · S. I. S. Traoré, MD \\ Hôpital de District de Bogodogo, Ouagadougou, Burkina Faso
}


Tableau Fréquence de la pratique de l'oxymétrie pulsée et de la check-list de l' OMS dans les hôpitaux

\begin{tabular}{|c|c|c|c|}
\hline & & $\begin{array}{l}\text { Avant Lifebox } \\
n(\%)\end{array}$ & $\begin{array}{l}\text { Après Lifebox } \\
n(\%)\end{array}$ \\
\hline $\begin{array}{l}\text { Pratique de } \\
\text { l'oxymétrie } \\
\text { pulsée pendant }\end{array}$ & $\begin{array}{l}\text { Nombre d'hôpitaux qui la } \\
\text { pratique de façon } \\
\text { non systématique }\end{array}$ & $15(26)$ & $0(0)$ \\
\hline l'anesthésie & $\begin{array}{l}\text { Nombre d'hôpitaux qui la } \\
\text { pratique systématiquement }\end{array}$ & $\begin{array}{l}42(74) \\
P=0.0003\end{array}$ & $57(100)$ \\
\hline $\begin{array}{l}\text { Pratique de la } \\
\text { Check-list de }\end{array}$ & $\begin{array}{l}\text { Nombre d'hôpitaux qui ne la } \\
\text { pratique pas }\end{array}$ & $47(83)$ & $18(32)$ \\
\hline l'OMS & $\begin{array}{l}\text { Nombre d'hôpitaux qui la } \\
\text { pratique } \\
P<0.0001 \\
\text { OMS = Organisation Mondiale de la Santé. }\end{array}$ & $10(17)$ & $39(68)$ \\
\hline
\end{tabular}

hôpitaux. La check-list de l'OMS était pratiquée dans 39 (68 \%) hôpitaux et de façon systématique par $23 \%$ des hôpitaux qui la pratiquaient. Pour améliorer cette pratique, $64 \%$ des enquêtés ont suggéré de former tous les membres de l'équipe chirurgicale.

Au Burkina Faso, avant la réalisation du projet Lifebox, $80 \%$ des salles d'opération dans les hôpitaux publics n'avaient pas d'oxymètre de pouls fonctionnel. Environ 1 an après la mise en œuvre de ce projet, nous avons constaté une amélioration significative de l'utilisation de cet équipement en per et postopératoires, donc de la surveillance des patients car la fréquence de pratique de l'oxymétrie était passée de 73 à $100 \%$ au cours de l'anesthésie et de 17 à $94 \%$ pour la surveillance du réveil.

La saturation du sang en oxygène est un paramètre de surveillance obligatoire ${ }^{1}$ mais au Burkina Faso l'utilisation systématique des oxymètres de pouls était encore faible en SSPI pour de nombreuses raisons : la rareté des SSPI dans les blocs opératoires; l'indisponibilité des oxymètres de pouls qui sont utilisés dans les salles d'intervention et la non perception de la nécessité de pratiquer l'oxymétrie de pouls en postopératoire immédiat chez les patients qui bénéficiaient d'une anesthésie locorégionale sans complication peropératoire et qui représentaient une très grande partie des interventions.
Quant à la pratique de la check-list au Burkina Faso, elle avait presque quadruplé suite à la formation Lifebox. Ce changement de pratique était important mais devrait être encore amélioré. L'amélioration de cette pratique nécessite l'implication de tous les acteurs du bloc opératoire et des premiers responsables des hôpitaux, ainsi que l'engagement des autorités sanitaires.

Le projet Lifebox a eu un impact positif significatif sur la pratique de l'oxymétrie pulsée et celle de la check-list de l'OMS dans nos hôpitaux publics au Burkina.

Conflit d'intérêt Aucun déclaré.

Responsabilité éditoriale Cet article a été traité par le Dr. Étienne de Médicis, Rédacteur de la langue française, Journal canadien d'anesthésie.

\section{Reference}

1. Société Française d'Anesthésie et de Réanimation. Décret sécurité 8/12/1994. Décret $n^{\circ} 94-1050$ du 5 décembre 1994 relatif aux conditions techniques de fonctionnement des établissements de santé en ce qui concerne la pratique de l'anesthésie et modifiant le code de la santé publique (troisième partie : Décrets). Disponible à l'adresse URL: http://sfar.org/decret-securite-8121994/ (consulté en août 2018). 